\title{
PER UNA STORIA DI DUE SECOLI DEL LOMBARDO E DI 150 ANNI DEL POLITECNICO
}

\author{
ANDREA SILVESTRI (*)
}

SuNTO. - La nascita, più di duecento anni fa, dell'Istituto Lombardo Accademia di Scienze e Lettere, e la fondazione centocinquanta anni fa del Politecnico di Milano si realizzarono in momenti storici di importanza capitale: la prima in epoca napoleonica (sul modello dell'Istituto nazionale che nella Francia rivoluzionaria aveva sostituito le soppresse Accademie), la seconda in prossimità dell'Unità d'Italia, secondo i più avanzati esempi europei di scuole politecniche. Queste origini così connotate, significative anche solo simbolicamente, si sarebbero proiettate da una parte nell'eccellenza scientifica e nell'impatto culturale dell'Istituto Lombardo, dall'altra nel radicamento del Politecnico nei grandi circuiti europei dell'istruzione superiore e dell'innovazione tecnico-scientifica. Il fatto che nell'Istituto Lombardo avessero spazio da subito gli interessi (e i premi) per le "utili invenzioni” spiega la presenza di un suo Gabinetto Tecnologico ricco di collezioni di macchine e strumenti. All'avvio del Politecnico, nell'ambito delle collaborazioni con Enti sinergici quali a Brera (oltre all'Istituto Lombardo) l'Osservatorio astronomico e l'Accademia di belle arti, e altrove la Società d'Incoraggiamento d'Arti e Mestieri e il Museo civico di storia naturale - il Gabinetto Tecnologico sarebbe stato acquisito dal Politecnico, anche per quella contiguità istituzionale con personaggi quali Francesco Brioschi e Giuseppe Colombo, fondatori e primi direttori del Politecnico, ma anche presidenti del Lombardo. Così come, del resto, le due storie (e i loro uomini) continuarono a procedere appaiate: dalle grandissime personalità della Destra storica appena citate, via via al fascismo (il rettore Gaudenzio Fantoli, o il ministro Giuseppe Belluzzo, poi presidente del Lombardo), alla Liberazione e alla ricostruzione post-bellica (per esempio, il rettore Gino Cassinis, più tardi sindaco di Milano), fino all'oggi: in un rapporto biunivoco di eccellenza e sobrietà, di responsabilità e competenze.

$* * *$

ABSTRACT. - The focus of this essay is on the relationship in Italy between scientific and technical culture on the one hand and "economic Risorgimento" on the other hand (the

(*) Istituto Lombardo Accademia di Scienze e Lettere, Politecnico di Milano, Italia. E-mail: andrea.silvestri@polimi.it 
latter to be understood as the condition and the driving force of the "political Risorgimento"). In the first part this relationship is shown to be linked to the more general idea of bringing Italy back into "modern European civilization", which in the first half of the XIX century was expressed both in heightened technological innovation and production, and in the rise of national movements for emancipation and independence. In the second part of the essay the measures are considered which were taken by the ruling classes after Unification in the face of remarkable difficulties and resistances. Thanks to such measures, the cultural conditions were created which enabled the subsequent industrial take-off between the XIX and XX century.

1. Carlo Emilio Gadda, uno dei più grandi scrittori del nostro Novecento, nei materiali preparatori dell'Adalgisa, intitolati Un fulmine sul 220 (titolo ben politecnico!), si ricorda sia appunto del Politecnico sia dell'Istituto Lombardo, contrapponendoli - con ironia - nel cuore di Donna Eloisa Bertolazzi, «la moglie del famoso Bertolazzi Membro dell'Istituto Lombardo [...] protofisico e primo elettromeccanico di Lombardia». Donna Eloisa è combattuta tra il ruolo di «nobildonna irraggiungibile, che è moglie, per giunta, di un Membro dell'Istituto Lombardo», e la «simpatia» ${ }^{1}$ per un giovane laureato del Politecnico di Milano, nato da una stirpe (i Cavigioli, poi Caviggioni) «con il bernoccolo dell'ingegnere $»^{2}$ e in dimestichezza con il «compianto senator ingegner Giuseppe Colombo [...] l'immortale autore del Manuale Colombo». Ma per sfortuna di Donna Eloisa «Lottare contro le virtù politecniche dei Cavigioli era impossibile: era più difficile che deragliare un tram» ${ }^{3}$.

Se Gadda era davvero di una famiglia politecnica, allievo e laureato in Ingegneria elettrotecnica, in giro per anni come ottimo professionista in Italia, in Europa e in Sudamerica con le sue proprie «virtù politecniche» invano contrapposte non a Donna Eloisa ma alla passione per la letteratura, Gadda - dicevo - non apparteneva al Lombardo, ma nelle sue opere tracce del Lombardo (non solo questa che ho citato) sono presenti come di un'istituzione gloriosa, profondamente radicata nella città, e simbolo - alla stessa maniera del Politecnico - di Milano,

1 Le citazioni che precedono provengono da C.E. Gadda, Un fulmine sul 220, a cura di Dante Isella, Milano, Garzanti, 2000, pp. 45-46.

2 Questa citazione e la seguente sono desunte da I ritagli di tempo, nell' Adalgisa (Opere di Carlo Emilio Gadda, a cura di D. Isella, Milano, Garzanti, 1988), p. 409.

3 Ancora da Un fulmine sul 220, cit., p. 46. 
della sua eccellenza, delle sue virtù: non senza, ripeto, punte di sarcasmo per certi conformismi di una società (secondo Gadda) più laboriosa che davvero colta ${ }^{4}$.

2. Non mi è possibile, naturalmente, ripercorrere la storia dei più di 200 anni dell'Istituto Lombardo e dei 150 del Politecnico. I brevissimi lineamenti che ne fornirò servono solo a consentire di tener dietro a incroci, passaggi di testimone, scambi di personalità e di ruoli, in epoche diverse e fondamentali: che vanno, tanto per schematizzare, dai tempi di Napoleone al Risorgimento, dall'Unità al fascismo, dall'antifascismo alla Liberazione, dalla ricostruzione postbellica al boom economico, dal '68 agli anni di piombo, eccetera eccetera. Per approfondimenti specifici rimando ai tre volumi della Storia dell'Istituto Lombardo $o^{5}$, ai tre volumi (a cavallo tra Lombardo, Politecnico e tanto altro) sul fondatore Francesco Brioschi $1_{185}{ }^{6}$, alla Storia del Politecnico di Milano di Ferdinando Lori ${ }_{1931}{ }^{7}$, al volume Electa sui primi cinquant'anni del Politecnico ${ }^{8}$ (Fig. 1), ai due tomi Cariplo-Laterza sul successivo mezzo secolo', e ora al libro per i centocinquant'anni dell'impatto del Politecnico sulle classi dirigenti del paese $^{10}$, più qualcosa di specifico che menzionerò in seguito.

3. Vengo dunque all'Istituto Lombardo. Dopo la Rivoluzione Francese, entro le repubbliche "giacobine" italiane fu importato da Parigi - sospesi i lavori delle vecchie, numerose ed eterogenee accademie

4 Fondamentale biografia, in verità non limitata a Infanzia e giovinezza di Gadda come reca il sottotitolo, è quella di G.C. Roscioni, Il Duca di Sant'Aquila, Milano, Mondadori, 1997.

5 Cfr. E. Gatti $1_{1969}$, G. Orlandi $1_{1985}$, A. Robbiati Bianchi e M. Vitale ${ }_{1963}$ (a cura di), L'Istituto Lombardo Accademia di Scienze e Lettere (Secoli XIX - XX), 3 voll., Milano, Istituto Lombardo Accademia di Scienze e Lettere - Libri Scheiwiller, 2007, 2008, 2009.

6 C.G. Lacaita, A. Silvestri 2006 (progetto editoriale di), Francesco Brioschi e il suo tempo (1824-1897), 3 voll. (I Saggi, II Inventari, III Scritti e discorsi), Milano, Franco Angeli, 2000, 2000, 2003.

7 Ferdinando Lori, Storia del R. Politecnico di Milano, Milano, Cordani, 1941.

8 Il Politecnico di Milano 1863-1914, Milano, Electa, 1981.

9 Il Politecnico di Milano nella storia italiana (1914-1963), Milano-Bari, Cariplo-Laterza, 1988.

10 C. Carboni, M. Granata, S. Morosini, G. Paletta, O. Selvafolta, A. Silvestri, F. Trisoglio (a cura di), Il Politecnico di Milano e la formazione delle classi dirigenti nazionali e locali, Cinisello Balsamo, Silvana Editoriale, 2013. 


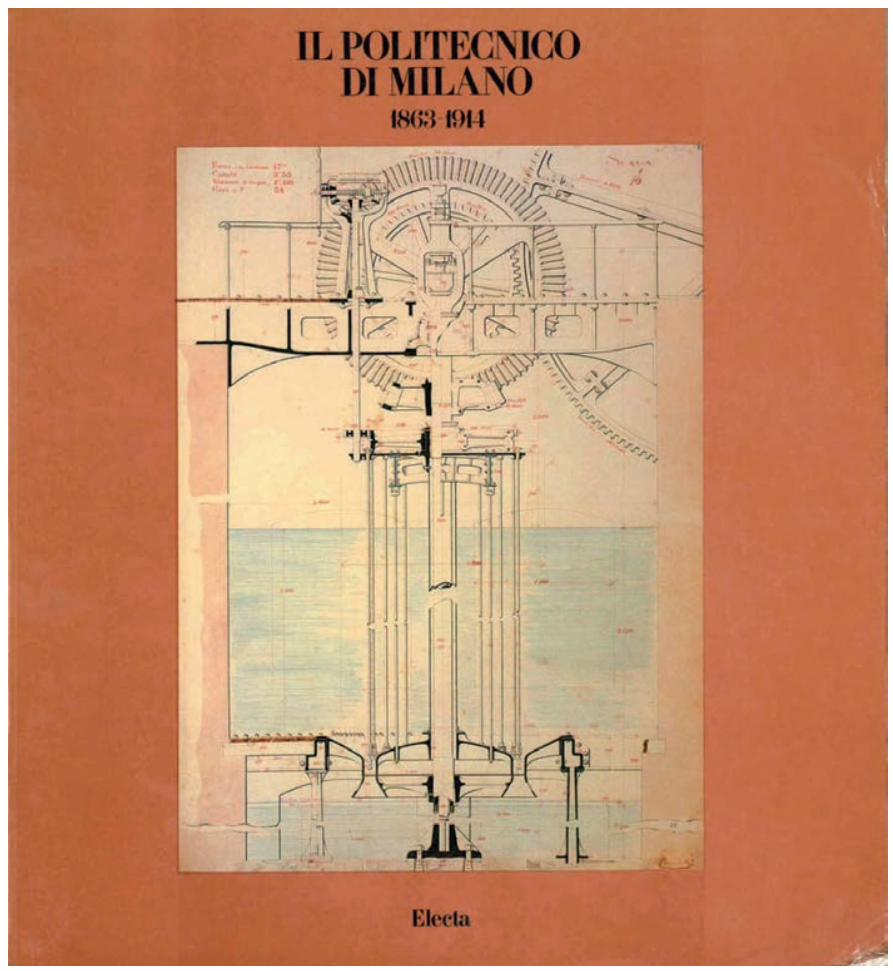

Fig. 1.

- il modello dell'Istituto nazionale. Nella Cisalpina prima, nella Repubblica italiana poi, infine nel Regno d'Italia, la sede dell'Istituto nazionale dal 1797 era Bologna, con crescente avversione dei lombardi che preferivano Milano, tra l'altro capitale del Regno, magari perfino secondo la soluzione di uno sdoppiamento della sede: sdoppiamento proposto da una commissione dell'Istituto presieduta da Alessandro Volta ${ }_{1802}$ dell'Università di Pavia, il «Franklin italiano» come lo si definiva nella nomina a membro dell'Istituto. Ma l'ipotesi fu respinta e l'attività dell'Istituto a Bologna continuò a languire, anche perché i membri di Milano e Pavia disertavano le sedute creando problemi di numero legale.

Napoleone (membro onorario nel 1803), convinto che «In Francia tutto è Parigi; in Italia non tutto è Milano» (1808), «Non è Milano nel 
Regno d'Italia ciò che è Parigi nell'Impero francese» $(1810)^{11}$, prima temporeggiò, e poi sembrò decidere a favore di uno smembramento fra Milano, Venezia, Bologna, Padova e Verona. Finalmente un decreto del dicembre 1810 introduceva la nuova denominazione di Istituto reale di scienze lettere ed arti, stabiliva la sede centrale a Milano e altrove le quattro sezioni; e insieme razionalizzava il ricco circuito culturale nel regno (accademie, società scientifiche o letterarie di Ancien régime) con l'istituzione di Atenei da coordinarsi con le sezioni e con l'Istituto reale. Così nel maggio 1811 iniziava qui a Brera l'attività dell'Istituto, che procedeva alla nomina dei "dotti" e al regolamento. Contemporaneamente l'Istituto era coinvolto dai governi napoleonici in incarichi di interesse pubblico, in particolare "premi d'industria" per "utili scoperte" nell'agricoltura e nelle arti. D'altra parte non mancò la partecipazione dell'Istituto, come negli anni bolognesi, a progetti in onore di Napoleone; il quale invece, nel giro di poco più di un anno, sarebbe precipitato verso la tragica conclusione in Russia.

4. Con il rientro degli Asburgo le autorità austriache furono naturalmente sospettose anche con l'Istituto, ribattezzato Imperialregio Istituto di scienze, lettere ed arti del Regno Lombardo-Veneto. Mentre le sezioni di Bologna e quelle venete erano in sofferenza, a Milano si cercava di stabilire buoni rapporti con le restaurate autorità, aderendo per esempio tempestivamente alla cancellazione di simboli e ricordi di Napoleone, a partire dal grande busto ora nella Sala delle adunanze. Rilievo nel lavoro accademico continuavano ad avere $\mathrm{i}$ premi, graditi agli Asburgo sia perché di tradizione teresiana sia per attenuare la conflittualità della transizione con uno sviluppo produttivo che desse benefici economici e sociali.

Significativa dal nostro punto di vista la decisione già nel 1815 di acquisire i modelli e i disegni delle apparecchiature premiate, per esporli al pubblico in particolari occasioni: primo nucleo del Gabinetto meccanico tecnologico il cui collegamento con il futuro Politecnico è già qui intuibile.

Un maggior interesse governativo per l'Istituto coincise con la per-

11 Cfr. E. Gatti, G. Orlandi, A. Robbiati Bianchi e M. Vitale (a cura di), L'Istituto Lombardo Accademia di Scienze e Lettere (Secoli XIX - XX), I. Storia Istituzionale, cit., pp. 66 e 69. 
cezione di Vienna di dover allentare la stretta repressiva e recuperare consenso, e servì per dare all'Istituto nuovo assetto, nuova composizione e sostegno finanziario. Circa i membri, Alessandro Manzoni ${ }_{1839}$ non accettò la nomina forse perché compromissoria con il regime austriaco, mentre nel campo scientifico menziono il nome di due nuovi "dotti" che hanno a che fare con le origini del Politecnico, in particolare con il suo fondatore Francesco Brioschi: Gabrio Piola ${ }_{1828}$, matematico "privato" a Milano, e Antonio Bordoni 1839 , professore di Matematica a Pavia (Fig. 2).

5. Nel trentennio che precede il '48, ricordo l'interesse dell'Istituto per i problemi agrari anche in campo educativo, e più in generale per il censimento della geologia dei siti, dei manufatti e delle produzioni, per le macchine a vapore, per la sicurezza dagli infortuni,

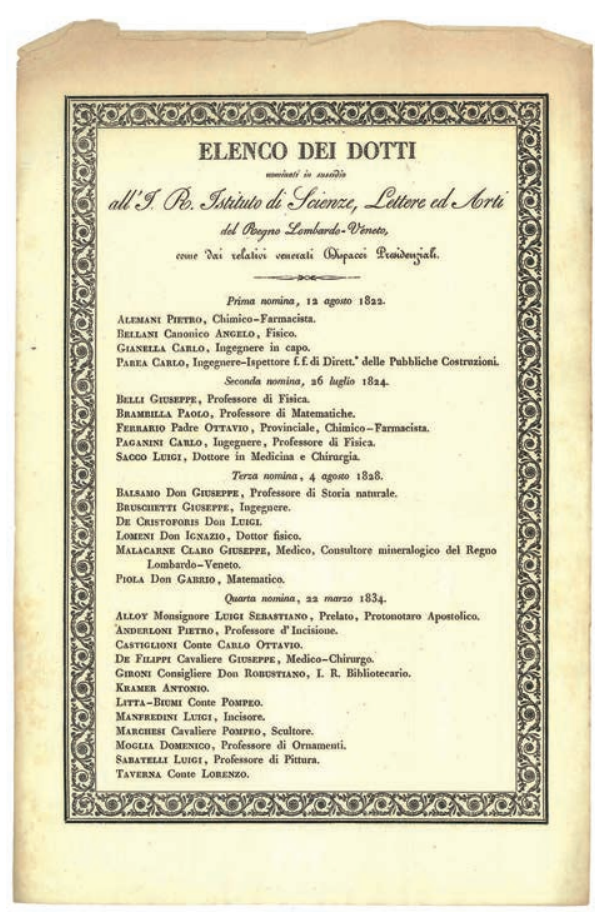

Fig. 2. 
per la piaga del lavoro minorile (Fig. 3). Circa la grande novità dell'illuminazione a gas, famosa nel 1819 l'ispezione dell'Istituto, conclusa positivamente, a casa di Luigi Porro Lambertenghi che aveva acquistato a Londra e installato privatamente un macchinario ad hoc: lo scopo di Porro Lambertenghi di ottenere una privativa fu ostacolata dal suo esilio dopo la congiura antiaustriaca del 1820-'21, ma la strada anche di questa innovazione e industrializzazione era aperta, e nel 1845 il centro di Milano sarebbe stato illuminato a gas ${ }^{12}$.

La raccolta museale del Lombardo diventa significativa - oltreché per incrementi di collezioni varie - quando si stabilizza un finanziamento specifico e trova una collocazione in insufficienti locali di Brera, dove Piola alla fine degli anni '40 lamenta non solo l'accumulo per ragioni di
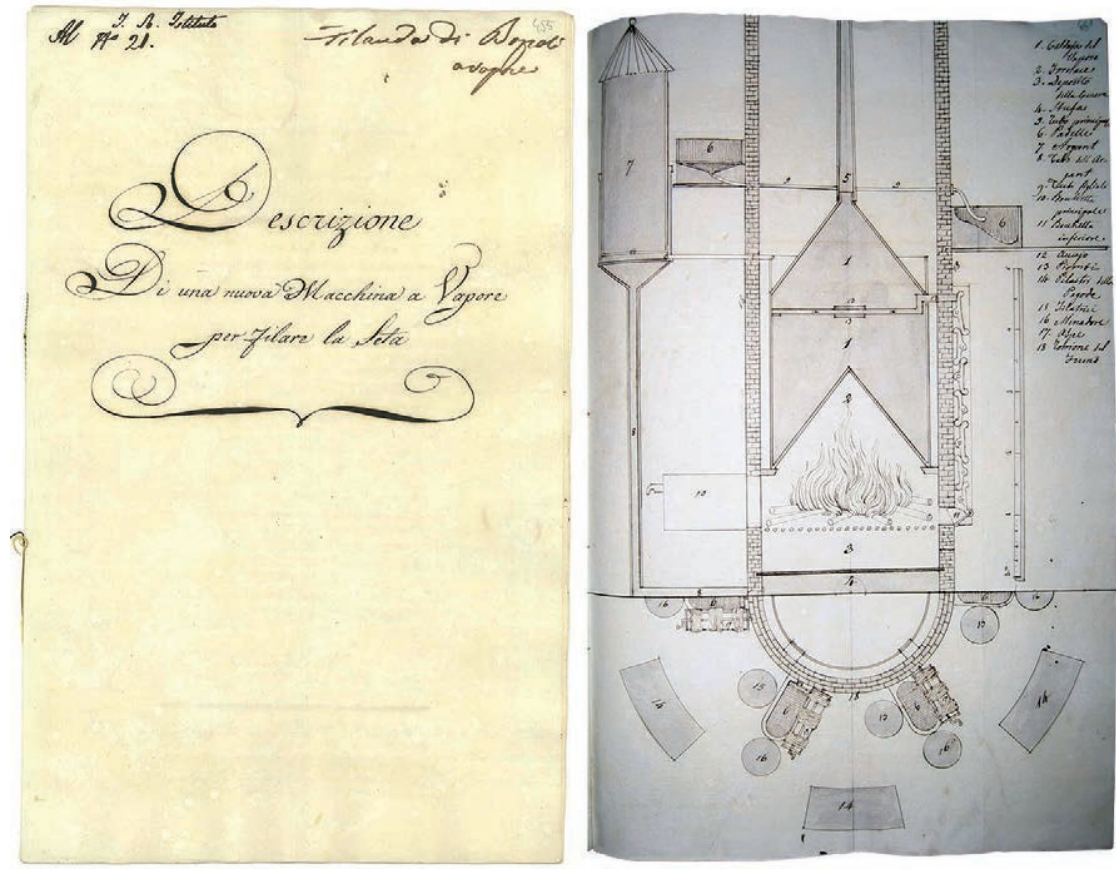

Fig. 3.

12 Cfr. la Storia istituzionale dell'Istituto Lombardo, cit., pp. 236-240. 
spazio, ma anche confusione e disordine, tanto più intollerabili vista la pur saltuaria apertura al pubblico ${ }^{13}$ (Fig. 4). Nuovo impulso al gabinetto viene dalla sesta riunione annuale degli scienziati italiani a Milano nel $1844^{14}$ (dopo l'avvio della serie a Pisa nel 1839, nonostante l'occhiuta attenzione granducale anche per il riferimento all'italianità). Per l'occasione l'Istituto nomina un'apposta commissione presieduta da Antonio de Kramer ${ }_{1839}$, chimico e industriale di nascita e con esperienze internazionali, esponente del più illuminato mondo produttivo, sul quale tornerò. Anche per un'iniziativa personale di Kramer sono acquisite nuove

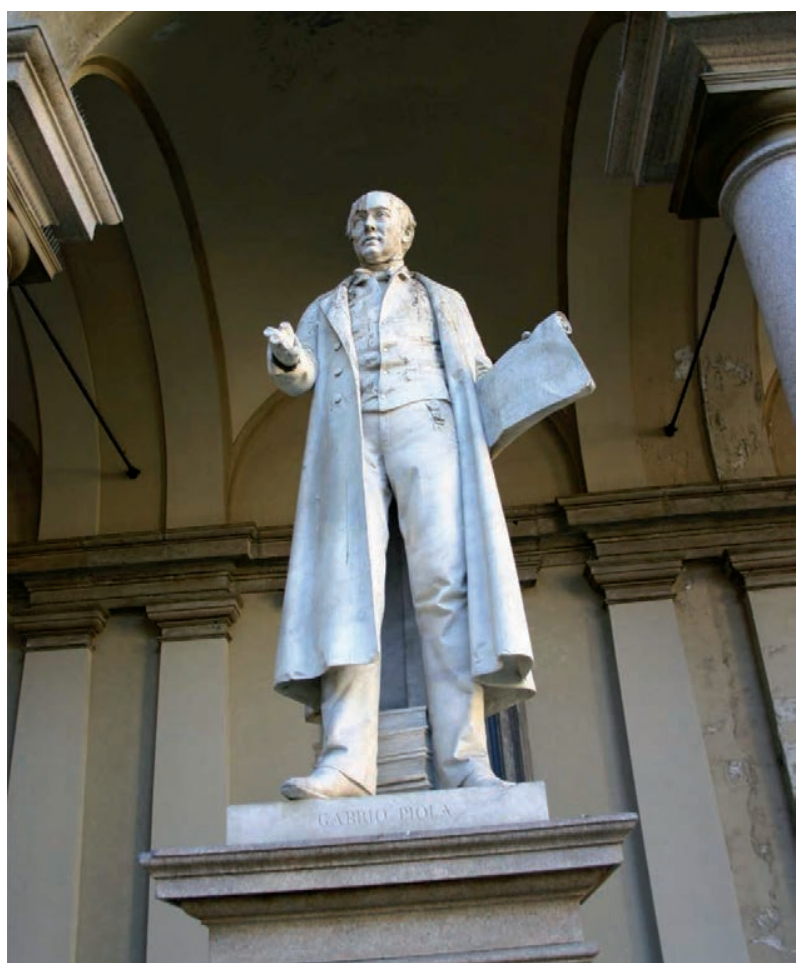

Fig. 4. Gabrio Piola (1794-1850).

13 Ib., p. 258.

14 Cfr. la recentissima edizione delle Notizie naturali e civili su la Lombardia, 2 tomi, a cura di Giorgio Bigatti, Le Monnier-Casagrande, Firenze-Bellinzona, 2014. 
macchine e strumenti sia per esigenze di studio del corpo accademico ${ }^{15}$, sia per soddisfare incarichi governativi, per es. sull'espansione tecnologica ed economica della Lombardia grazie allo sfruttamento di forze motrici idrauliche e a vapore. Ma i risultati della commissione non sono del tutto soddisfacenti, forse anche per «qualche frizione con alcuni dei membri» dell'Istituto ${ }^{16}$; tuttavia nel 1846 si arriva a un catalogo a stampa del gabinetto che è anche, quasi, una guida museale in giro per le poche stanze.

6. Già che ho parlato di Kramer, ricordo che nel 1838 (lo stesso anno dello smembramento dei due istituti di Milano e Venezia) era stata fondata, dalla Camera di commercio milanese e dai ceti imprenditoriali lombardi più avvertiti, la Società d'incoraggiamento d'arti e mestieri, allo scopo di "migliorare le arti utili e le manifatture" ${ }^{17}$. Tra i fondatori c'è proprio Kramer e altri personaggi partecipi pure dell'Istituto lombardo. Dopo pochi anni la Società d'incoraggiamento avvia i primi corsi e laboratori di una disciplina particolarmente arretrata in Italia, la Chimica; finché nel 1845 entra a farne parte Carlo Cattaneo ${ }_{1843}$, (Fig. 5) reduce dalla prima grandiosa serie della sua rivista "Il Politecnico", e da poco nominato membro dell'Istituto: Cattaneo porta al suo nuovo ruolo di relatore della Società d'incoraggiamento la propria esperienza di conoscitore e promotore della modernizzazione del paese. In una commissione del Lombardo sull'estensione del perimetro d'azione della Società d'incoraggiamento da Milano alle altre province lombarde, Cattaneo sconsiglia dal proseguire sulla strada dispersiva e ripetitiva dei premi industriali. Nella direzione cattaneana dell'avanzamento culturale, anzi dell' "incivilimento", la Società d'incoraggiamento dopo il '48 avrebbe quindi spostato i propri obiettivi verso l'istituzione di scuole (di Chimica, di Meccanica e disegno, di Setificio) dotate di laboratori e volte all'istruzione tecnica di operai, operai specializzati, quadri intermedi: quasi un incunabolo del futuro Politecnico, non a caso debitore di sinergie alla Società d'incoraggiamento e all'Istituto Lombardo, così come ad altri Enti di cui dirò.

15 Cfr. la Storia istituzionale dell'Istituto Lombardo, cit., p. 262.

16 Ib., p. 265.

17 C.G. Lacaita, L'intelligenza produttiva. Imprenditori, tecnici e operai nella Società d'Incoraggiamento d'Arti e Mestieri di Milano (1838-1988), Milano, Electa, 1990. 


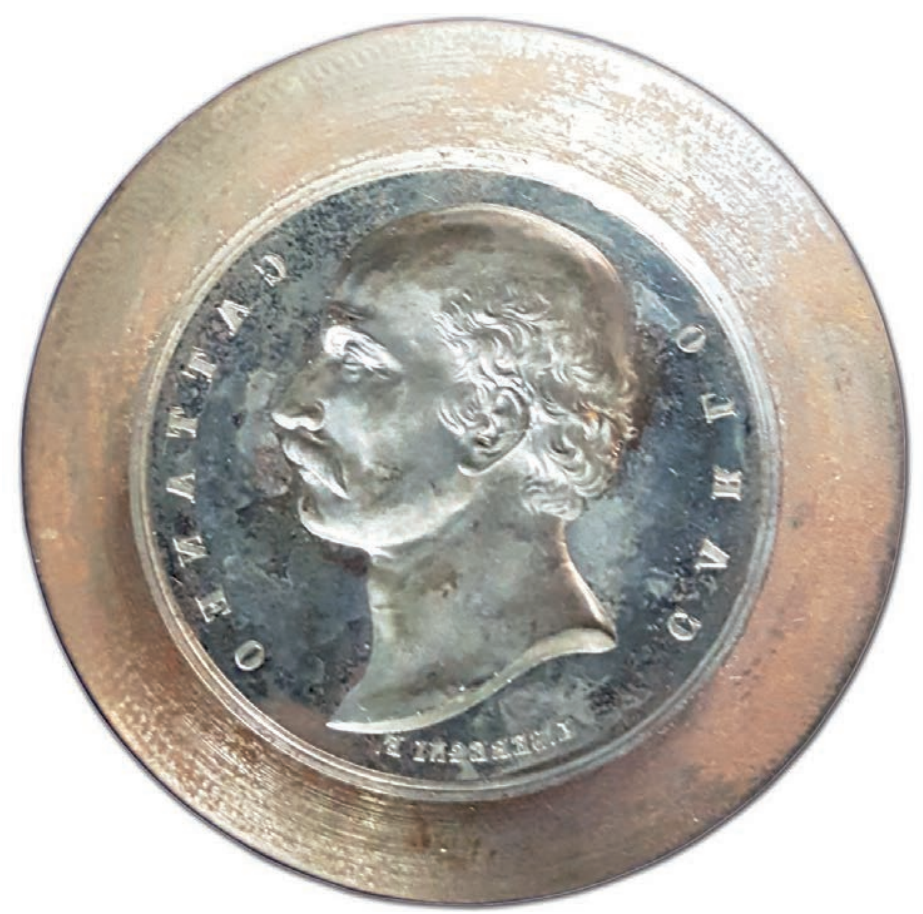

Fig. 5. Punzone della medaglia commemorativa dedicata a Carlo Cattaneo di L. Seregni, dal bassorilievo di G. Strazza, 1871 (Arch. Istituto Lombardo).

7. Subito prima delle Cinque giornate, un'altra commissione dell'Istituto, richiesta dal governo in un tentativo estremo di riavvicinamento con i governati, su un progetto di riforma dell'istruzione in Lombardia, ebbe come relatore ancora Cattaneo. Nel documento si trovano proposte molto innovative su ogni ordine di scuole: tra le quali ricordo la sottolineatura della necessità generalizzata di cultura tecnica, e in ambito universitario l'auspicio di un corso di perfezionamento per gli ingegneri, da stabilirsi a Milano, in una città industriale capace di raccordare l'impostazione teorica degli allievi ingegneri-architetti nel corso matematico a Pavia con una visione più pratica. Il progetto fu travolto dalla rivoluzione, ma qualche segno ne resterà alla nascita del 
Politecnico ${ }^{18}$, più di quanto lasci intravvedere la polemicità di Cattaneo contro Gabrio Casati (capo moderato e filo sabaudo delle Cinque giornate e poi nel 1859 autore a Torino dell'omonima legge istitutiva dei Politecnici di Torino e Milano), e contro il fondatore di quello di Milano Francesco Brioschi e la consorteria milanese.

La partecipazione del Lombardo alla rivoluzione del '48 fu condivisa pur con varia intensità, e la ripresa al rientro degli Asburgo fu lenta e difficile per la durezza del regime autoritario imposto: a partire dall'epurazione dei suoi uomini più esposti, tra cui Kramer e Cattaneo, o dall'indagine poliziesca sulle opinioni politiche e sui comportamenti degli associati al Lombardo. Nel decennio 1850-1859 segnalo la persistente prevalenza nei premi e nelle pubblicazioni di tematiche tecnicoproduttive, con spazio ridotto per le discipline umanistiche; e ricordo specificatamente tre fatti:

- non viene meno l'interesse per il gabinetto tecnologico così come per la Biblioteca dell'Istituto, tant'è che nel 1852 sono istituiti due regolari "conservatori" ${ }^{19}$ che sovraintendono un lento ma costante incremento delle dotazioni;

- nel 1854 è nominato membro effettivo il matematico-fisico Giovanni Codazza $_{1833}$ di Pavia, che nel '48 era stato rettore, e che nel '63 si trasferirà con Brioschi al Politecnico di Milano per insegnare la Fisica tecnologica, passando dopo pochi anni al Museo industriale di Torino, dove fu maestro di Galileo Ferraris;

- nel breve governatorato generale del Regno, dal '57, di Massimiliano d'Asburgo (membro onorario nel 1856), fratello di Francesco Giuseppe, è elaborato, soprattutto da Cesare Cantù 1854 , segretario del Lombardo e vicino a Massimiliano, un progetto per l'istruzione, che in particolare prospetta la creazione di una nuova scuola politecnica secondo gli esempi già rodati nell'Impero: ma Vienna blocca ogni riforma.

8. Nel 1859, al termine della Seconda guerra d'Indipendenza, la Lombardia è annessa al Piemonte: tra i primi atti, il re Vittorio Emanuele

18 Cfr. A. Silvestri, Il progetto di riforma scolastica dell'Istituto Lombardo (1848, relatore Carlo Cattaneo) e la Legge Casati (1859), in «Rendiconti», n. 145, Milano, Istituto Lombardo - Accademia di scienze e lettere, 2012.

19 Cfr. la Storia istituzionale dell'Istituto Lombardo, cit., p. 423. 
II il 15 giugno nomina Manzoni "presidente perpetuo" dell'Istituto, Istituto che partecipa pienamente alle nuove prospettive unitarie. Il 13 novembre 1859 è promulgata (ancora in regime di pieni poteri) la Legge Casati sull'intero settore dell'Istruzione pubblica, con in campo tecnico le due grandi novità dei cosiddetti "stabilimenti tecnici" secondari e delle prime due Scuole superiori per gli ingegneri a Torino e a Milano.

A Milano risulta determinante il lavoro di Francesco Brioschi, giovane professore alla Facoltà matematica di Pavia (in pratica una scuola di ingegneria-architettura): dove nel '61 Brioschi - proprio mentre preparava a Milano la partenza del Regio Istituto Tecnico Superiore - sarà anzi brevemente rettore dell'Università. Brioschi era stato allievo del già citato Antonio Bordoni, maestro appassionato di tanti altri protagonisti della prima generazione di professori al Politecnico nonché tutti membri del Lombardo: Colombo ${ }_{1862}$, Codazza, i matematici Felice Casorati ${ }_{1865}$, Luigi Cremona 1864 e Eugenio Beltrami ${ }_{1868}$, l'agronomo Carlo Pasi ${ }_{1857}$, lo scienziato delle costruzioni Celeste Clericetti $1_{1872}$.

La prima memoria scientifica di Brioschi, Sul moto del calore nel globo della terra («un confronto, scriverà Luigi Amerio ${ }_{1931^{20}}$, tra le impostazioni di Fourier e Poisson»), gli era stata suggerita da Gabrio Piola, del cui domestico circolo matematico (più aperto verso la modernità matematica rispetto a Bordoni, nonostante lo spiritualismo di Piola) Brioschi era entrato a far parte a Milano ereditando da Piola anche interessi idraulici. Il lavoro di Brioschi, ventitreenne, fu pubblicato grazie a Piola stesso sul "Giornale dell'Imperial Regio Istituto LombardoVeneto e Biblioteca italiana" (non entro nelle vicende delle pubblicazioni periodiche dell'Istituto, rimandando a quanto ne ha scritto Franco Della Peruta 1999 nel primo volume della Storia istituzionale).

Brioschi, dopo aver partecipato al '48 ed essersi via via spostato su posizioni filo-sabaude, vivrà una carriera scientifica brillantissima, presto protesa verso l'internazionalità: il suo volume La teorica dei determinanti del '54 fu subito tradotto in francese e tedesco; e al '58 risale il suo viaggio con Casorati ed Enrico Betti ${ }_{1940}$ (dell'Università di Pisa, ma anche del Lombardo), in Svizzera, Germania e Francia.

Brioschi, che sarà per due volte presidente del Lombardo, così come presidente dell'Accademia dei XL, e poi dei Lincei subito dopo

20 Francesco Brioschi e il suo tempo (1824-1897). cit., I Saggi, a cura di C.G. Lacaita e A. Silvestri, Milano, Franco Angeli, 2000, p. 13. 
il rifondatore Quintino Sella ${ }_{1861}$ (socio dell'Istituto Lombardo), a partire dal '59 della Legge Casati deve lavorare molto sia a Torino sia a Milano per arrivare al decreto attuativo del suo Politecnico. Nella capitale, contro le sue pessimistiche previsioni, lo aiuta la sintonia con Quintino Sella ${ }^{21}$, al quale anzi subentra nella carica di Segretario generale della Pubblica istruzione. A Milano invece Brioschi deve coordinare la collaborazione con il nascente Politecnico di Enti potenzialmente sinergici ma sospettosi del dinamismo di Brioschi e gelosi della propria autonomia: intendo la Società d'Incoraggiamento, che volentieri mette a disposizione suoi professori di punta (Colombo in primis, ma lo stresso Brioschi...), suoi laboratori e gabinetti, ma è ben convinta di non doversi annullare nel Politecnico; intendo il Museo civico di storia naturale con le sue collezioni mineralogiche e geologiche, diretto da Emilio Cornalia ${ }_{1853}$, due volte presidente del Lombardo e futuro professore di Zoologia al Politecnico; e intendo a Brera l'Osservatorio astronomico, pure con le sue attrezzature e con i suoi docenti (Giovanni Schiaparelli $1_{1862}$ e poi Giovanni Celoria ${ }_{1873}$, entrambi presidenti del Lombardo); e intendo lo stesso Istituto lombardo, il cui Gabinetto tecnologico avrebbe potuto essere ceduto al Politecnico con i suoi oggetti "scientifici" e/o "tecnologici": i primi destinati a studi del corpo accademico e forse da escludere, i secondi più fruibili in una struttura di istruzione tecnica superiore e perciò alienabili ${ }^{22}$. Pare che siano stati in realtà poi acquisiti dal Politecnico gli uni e gli altri, anche se a oggi pur avendo fatto un censimento delle apparecchiature storiche superstiti $^{23}$ - non ho trovato nulla: e mi pare credibile che due trasferimenti di sede, soprattutto quello massiccio del 1927 dalla Canonica di San Bartolomeo in Piazza Cavour a Città Studi in Piazza Leonardo da Vinci, dopo 60 anni di vita sempre più gravata da coabitazioni ${ }^{24} \mathrm{e}$ da problemi di spazio, possano aver fatto perdere questo tesoro storico e museale.

21 Cfr. il mio lavoro Il contributo [di Sella] all'avvio del Regio Istituto Tecnico Superiore di Milano, in Quintino Sella scienziato e statista per l'Unità d'Italia, Roma, Scienze e Lettere, 2013.

22 Cfr. A. Castellano, Le relazioni tra il Politecnico e la società del tempo (18631914), in Il Politecnico di Milano 1863-1914, cit., pp. 164-165.

23 A. Silvestri, Per ricordare e conoscere. Dai laboratori e dagli archivi del Politecnico, Milano, All'insegna del pesce d'oro di Vanni Scheiwiller, 1997.

24 A lungo con l'Accademia scientifico-letteraria di cui in 9., con il Collegio degli ingegneri e architetti, perfino con il CAI. 
9. Brioschi inaugura il Politecnico il 29 novembre 1863 insieme all'Accademia scientifico-letteraria, la futura Facoltà di Lettere e Filosofia quando nel 1924 sarà istituita l'Università degli Studi di Milano, allora "la Regia" oggi "la Statale". È il caso di sottolineare in questa duplice inaugurazione come secondo Brioschi «le scienze positive e gli studi letterari, storici e filosofici» non debbano essere soggetti a una «separazione [...] sempre funest $a$ al loro progresso» ${ }^{25}$. E tuttavia la preminenza di Brioschi (che fino alla morte sarà presidente dell'Accademia, così come direttore del Politecnico) faceva insorgere pubblicamente lo storico della lingua Graziadio Isaia Ascoli ${ }_{1862}$ direttore dell'Accademia stessa: il quale già in una seduta del Lombardo nel 1864 lamentava - contrastato da Brioschi - la dipendenza dell'Accademia dal Politecnico e dalla Scuola superiore d'Agricoltura (presieduta guarda caso da Brioschi), e indicava questa debolezza dell'Accademia come una "debolezza anche per l'Istituto lombardo". Proprio in questo giro d'anni, l'Istituto perde nella sua denominazione il riferimento finale alle "arti", perché le arti belle erano già da decenni passate alle competenze dell'Accademia di Brera, e le "arti utili" di cui parlava Cattaneo, e cioè le "arti tecnologiche, industriali, manifatturiere" ${ }^{26}$, gravitavano da quel momento nell'orbita del Politecnico.

Una delle novità del Politecnico, non dirò rispetto a Pavia, ma anche rispetto al Politecnico di Torino, era la specializzazione, che vedeva - accanto alla tradizionale sezione civile (praticamente sottratta all'Università ticinense) - una sezione assolutamente inedita in Italia, quella meccanica poi industriale, fortemente voluta da Giuseppe Colombo: assertore appunto dell'industrializzazione del paese, già nel suo manuale dell'ingegnere ricordato da Gadda e poi nell'attività imprenditoriale, editoriale, politica, Colombo sarebbe stato per tre volte presidente del Lombardo.

Due anni dopo l'inaugurazione del Politecnico, e cioè nel 1865, nasce la sezione di Architettura civile, risultato della collaborazione di Brioschi con Camillo Boito ${ }_{1893}$ (Fig. 6) dell'Accademia di Belle Arti:

25 C.G. Lacaita (a cura di), Francesco Brioschi e il suo tempo (1824-1897). III Scritti e discorsi, cit., p. 51.

26 A. Castellano, Le relazioni tra il Politecnico e la società del tempo (18631914), cit., p. 165. 


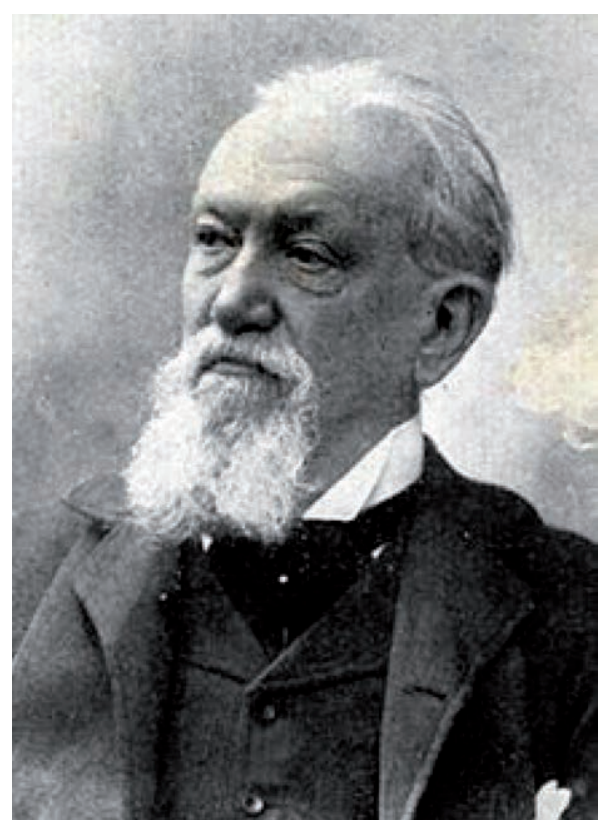

Fig. 6. Camillo Boito (1936-1914).

entrambi sono convinti che i due anni del diploma di Disegno architettonico che l'Accademia forniva (e per la verità avrebbe continuato contraddittoriamente a fornire) ai laureati della Facoltà matematica di Pavia dovessero essere sostituiti da un triennio politecnico di specializzazione, dove le discipline artistiche di Brera erano inserite in un più solido contesto tecnico-scientifico.

10. Colombo, successore di Brioschi alla direzione del Politecnico dal 1897 al $1921^{27}$, ai tempi della Grande Guerra ha un'evoluzione

27 Cfr. l'introduzione di C.G. Lacaita a G. Colombo, Industria e politica nella storia d'Italia. Scritti scelti: 1861-1916, a cura di C.G. Lacaita, Roma-Bari, CariploLaterza, 1985; di Colombo (e di Gadda) parla inoltre il mio saggio Il Politecnico di Milano e la Grande Guerra: due generazioni, due ingegneri, due esperienze a confronto, in P. Del Negro (a cura di), Le Università e le guerre dal Medioevo alla Seconda guerra mondiale, Bologna, CLUEB, 2011. 
(dall'antimilitarismo e anticolonialismo ai tempi di Crispi) a favore della guerra in Libia e quindi dell'interventismo: evoluzione che è analoga a quella del Lombardo. Invece il trapasso verso il fascismo, tra i problemi sociali e il travaglio delle "vittoria mutilata", è quasi sottaciuto e messo tra parentesi dall'Istituto, che preferisce concentrarsi sulle celebrazioni ora dantesca ora manzoniana ora voltiana, o sul contrastato trasferimento della Biblioteca di Brera a Palazzo Marino, o sull'Archivio di Stato di Milano. Questo tentativo di difesa dell'identità del Lombardo, sempre più attento peraltro ai valori della cultura umanistica, sembra inizialmente prescindere da presenze importati che al suo interno sono vicine al regime. Mi riferisco alla presidenza di un uomo di rilievo nel fascismo come Ulisse Gobbi $i_{1884}$, professore e poi rettore della Bocconi ma anche docente al Politecnico. Mi riferisco al fatto che era al Lombardo già dal 1906 Gaudenzio Fantoli ${ }_{1906}^{28}$ : dopo le brevi direzioni al Politecnico di Cesare Saldini ${ }_{1917}$ (socio corrispondente del Lombardo e membro effettivo il giorno successivo alla sua morte nel 1922), e dopo la contrastata direzione di un altro uomo del Lombardo, Luigi Zunini $_{1902}$, dal 1926 al 1940 Fantoli fu il direttore del Politecnico ai tempi del fascismo. E mi riferisco al fatto che era socio anche Giuseppe Belluzzo $_{1922}$, il quale nei secondi anni Venti sarebbe stato ministro con Mussolini ${ }^{29}$, e poi presidente del Lombardo.

Ma la fascistizzazione esplicita avanza anche al Lombardo: si passa dall'obbligo del giuramento al regime nel 1931, al regio decreto del 1935 che demanda la scelta delle cariche direttive anche dell'Istituto al ministro dell'Educazione nazionale. Successivamente, nel 1938, avviene l'espulsione dal Lombardo (essendo presidente in carica Giuseppe Bruni ${ }_{1923}$ (Fig. 7), professore di Chimica generale al Politecnico) di Giuseppe Antonio Borgese ${ }_{1924}$ della Statale, espulsione che già prima Fantoli aveva chiesta a Mussolini. Ma soprattutto si avviano nello stesso '38 le inique leggi razziali, puntualmente eseguite da Bruni (come da Fantoli al Politecnico, e come in altre istituzioni culturali $)^{30}$, con l'espulsione di venti tra membri e soci del Lombardo, illustri

28 C.G. Lacaita, I tecnici milanesi dal moderatismo al fascismo: il caso Fantoli, in Il Politecnico di Milano nella storia italiana (1914-1963), cit.

29 M. Minesso, Giuseppe Belluzzo. Tecnico e politico nella storia d'Italia 18761952, Milano, FrancoAngeli, 2012.

30 Cfr. A. Silvestri e S. Morosini, Le leggi razziali (1938) e l'Istituto Lombardo, in «Rendiconti dell'Istituto Lombardo Accademia di Scienze e Lettere», 143/2009, pp. 


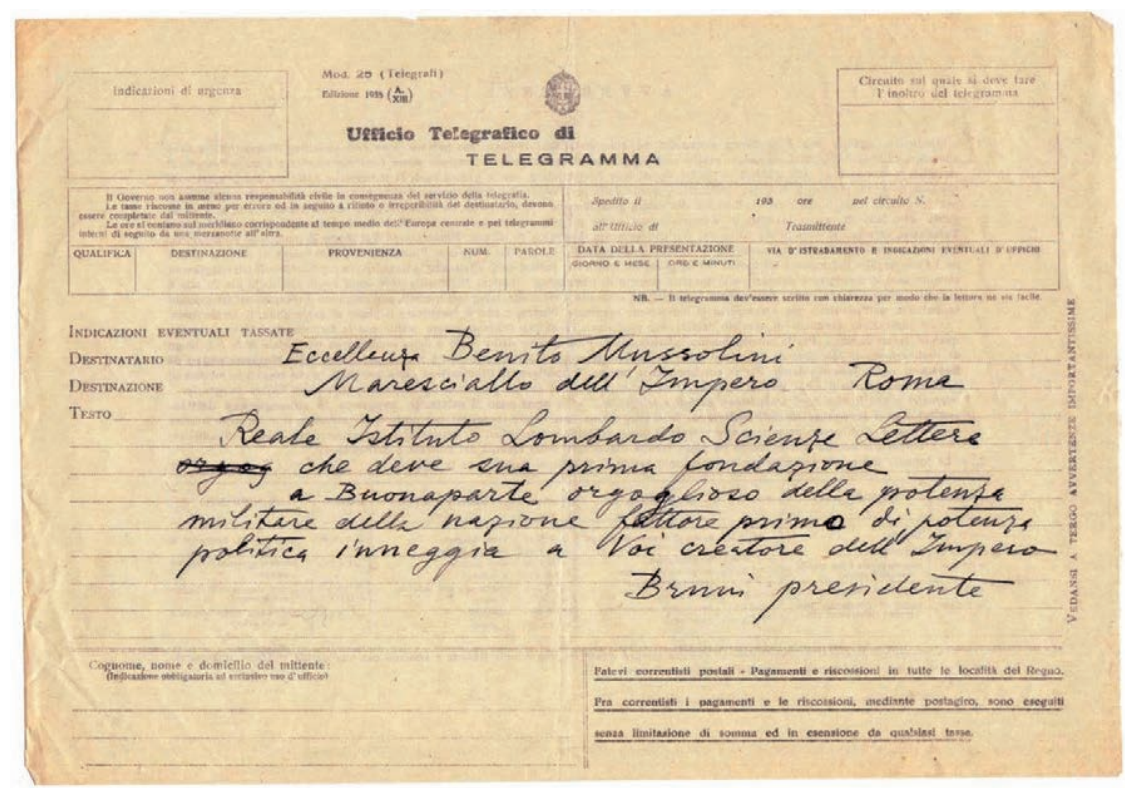

Fig. 7.

quando non geniali, più cinque personalità straniere tra cui Henry Bergson $_{1923}$ e Albert Einstein ${ }_{1922}$ : quest'ultimo però, tempestivamente, si era dimesso da tutte le istituzioni italiane colpite dai provvedimenti antiebraici ${ }^{31}$. Le sostituzioni al Lombardo, al contrario che altrove, non furono connotate dalla fede politica, e anzi dal ' 40 i Rendiconti ospitano due necrologie di ex-soci ebrei, con una progressiva presa di distanza dal regime.

La vita accademica dell'Istituto si fa sempre più precaria. Nel 1942 è nominato presidente Belluzzo, che nella seduta inaugurale auspica "una pace giusta e duratura", "rispetto fra i popoli", ma parla

9-35. Su altre istituzioni, in particolare l'Associazione elettrotecnica italiana, si cfr. S. Morosini, A settant'anni dell'espulsione dei soci ebrei dell' Associazione elettrotecnica italiana, in «AEIT», anno 2008, n. 12, pp. 52-55.

31 A. Capristo, L'espulsione degli ebrei dalle accademie italiane, Torino, Zamorani, 2002, p. 53. 
anche contro i "plutocratici imperialismi". La prima affermazione che ho appena ricordato, insieme ad altre prove, indurranno Belluzzo a contestare vibratamente la decadenza dopo la Liberazione - come dirò - sia dal Lombardo (come Bruni), sia dal Senato; mentre il suo riferimento agli imperialismi plutocratici sembra invece confermare la sua fedeltà al regime.

11. Anche il rettore della Liberazione e della ricostruzione postbellica al Politecnico, Gino Cassinis ${ }_{1338}$, (Fig. 8) faceva parte (come dei Lincei e dell'Accademia delle Scienze di Torino) del Lombardo dal 1938. Nel 1944, vicedirettore al Politecnico, è proposto come direttore da tutti i colleghi al ministro della Repubblica sociale Carlo Alberto Biggini, non entro una terna (come previsto dalla Repubblica di Salò) per la scelta del ministro, ma come unico nome, e il ministro accetta. Durante l'occupazione tedesca Cassinis sostiene con forza ma con prudenza la Scuola, che diventa un centro di cospirazione clandestina fino alla Liberazione, quando Cassinis è rieletto democraticamente all'unanimità dai professori del Politecnico, in un rettorato che procederà alla

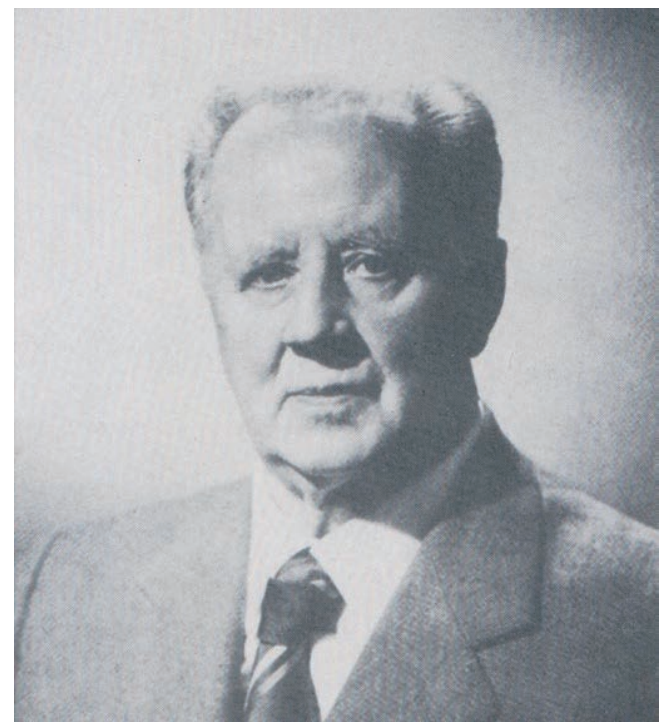

Fig. 8. Gino Cassinis (1885-1964). 
ricostruzione e al rinnovamento e che durerà fino al 1960. Successivamente, fino alla morte, sarà sindaco di Milano in un innovativo esperimento di centro sinistra ${ }^{32}$.

Con la Liberazione partiva la procedura di epurazione antifascista sia al Politecnico sia al Lombardo: al Politecnico la commissione era presieduta da Arturo Danusso ${ }_{1931}$, professore di Scienza delle costruzioni e membro del Lombardo, e anche qui Danusso fu proposto come membro della commissione dal Commissario del Lombardo Antonio Banfi $_{1933}$, il grande filosofo alla Statale. Mentre al Politecnico, sostanzialmente in nome della neutralità della tecnica, e in assenza di denunce scritte e firmate, la procedura finiva praticamente in nulla ${ }^{33}$, l'epurazione al Lombardo toccò cinque membri effettivi (tra cui, come già ricordato, Belluzzo e Bruni) e sei corrispondenti.

Non ho tempo naturalmente per percorrere la strada comune delle nostre due istituzioni in età repubblicana. Per venire a tempi vicino a noi, ma limitandomi a personaggi che ho avuto la fortuna di conoscere e ammirare, mi fa piacere ricordare almeno le presidenze del Lombardo di insigni Maestri e professori emeriti del Politecnico, Luigi Amerio, Emilio Gatti ${ }_{1969}$ e Giannantonio Sacchi Landriani ${ }_{1983}$.

32 M. Punzo, Gino Cassinis sindaco di Milano, in Il Politecnico di Milano nella storia italiana (1914-1963), cit.

33 Unico epurato al Politecnico fu Michele Lo Presti, allievo di Belluzzo, «che aveva aderito alla Repubblica sociale e che negli anni passati, mosso da un profondo amor di Patria, era stato un convinto sostenitore del regime fascista. Distrutto moralmente e fisicamente già dalla disfatta del Paese, egli non resse all'umiliazione del verdetto e si spense pochi giorni dopo» (Giovanni B. Stracca, La vita del Politecnico tra 1914 e il 1963. Eventi principali, attività istituzionali e dati statistici, nel vol. I di Il Politecnico di Milano nella storia italiana (1914-1963), cit., p. 68). 